\title{
Effects of Single and Combined Water, Sanitation and Handwashing Interventions on Fecal Contamination in the Domestic Environment: A Cluster-Randomized Controlled Trial in Rural Bangladesh
}

Ayse Ercumen, ${ }^{*}, \dagger \oplus$ Andrew Mertens, ${ }^{\ddagger}$ Benjamin F. Arnold, ${ }^{\ddagger}$ Jade Benjamin-Chung, ${ }^{\ddagger}$ Alan E. Hubbard, Mir Alvee Ahmed, ${ }^{\S}$ Mir Himayet Kabir, ${ }^{\S}$ Md. Masudur Rahman Khalil, ${ }^{\S}$ Ashish Kumar, ${ }^{\S}$

Md. Sajjadur Rahman, ${ }^{\S}$ Sarker Masud Parvez, ${ }^{\S}$ Leanne Unicomb, ${ }^{\S}$ Mahbubur Rahman, ${ }^{\S}$ Pavani K. Ram, Thomas Clasen, ${ }^{\perp}$ Stephen P. Luby, ${ }^{\#}$ and John M. Colford, Jr. ${ }^{\ddagger}$

${ }^{\dagger}$ Department of Forestry and Environmental Resources, North Carolina State University, Raleigh, North Carolina 27695, United States

${ }^{\ddagger}$ School of Public Health, University of California, Berkeley, California 94720, United States

${ }^{\S}$ Infectious Disease Division, International Centre for Diarrhoeal Disease Research, Bangladesh, Dhaka, 1212, Bangladesh

"University at Buffalo, Buffalo, New York 14214, United States

${ }^{\perp}$ Rollins School of Public Health, Emory University, Atlanta, Georgia 30322, United States

\#Infectious Diseases \& Geographic Medicine, Stanford University, Stanford, California 94305, United States

Supporting Information

ABSTRACT: Water, sanitation, and hygiene interventions have varying effectiveness in reducing fecal contamination in the domestic environment; delivering them in combination could yield synergies. We conducted environmental assessments within a randomized controlled trial in Bangladesh that implemented single and combined water treatment, sanitation, handwashing (WSH) and nutrition interventions (WASH Benefits, NCT01590095). After one and two years of intervention, we quantified fecal indicator bacteria in samples of drinking water (from source or storage), child hands, children's food and sentinel objects. In households receiving single water treatment interventions, Escherichia coli prevalence in stored drinking water was reduced by $50 \%$ and concentration

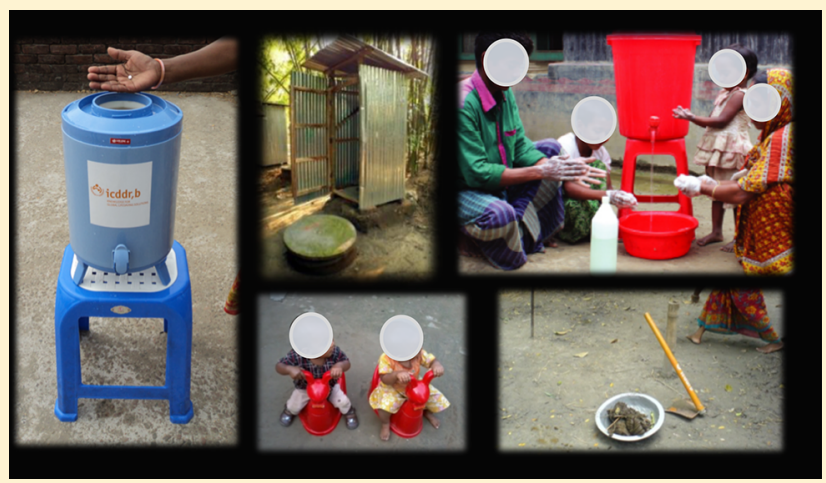
by 1 -log. E. coli prevalence in food was reduced by $30 \%$ and concentration by $0.5-\log$ in households receiving single water treatment and handwashing interventions. Combined WSH did not reduce fecal contamination more effectively than its components. Interventions did not reduce E. coli in groundwater, on child hands and on objects. These findings suggest that WSH improvements reduced contamination along the direct transmission pathways of stored water and food but not along indirect upstream pathways. Our findings support implementing water treatment and handwashing to reduce fecal exposure through water and food but provide no evidence that combining interventions further reduces exposure.

\section{BACKGROUND}

Diarrheal disease transmission occurs through a complex web of environmentally mediated pathways including drinking water, food, hands, fomites, and vectors. The complexity arises from the multitude of transmission routes, broad diversity of pathogens, importance of environmental conditions, and interactions between the environment and human behavior. Treating drinking water before consumption and washing hands with water and soap lower fecal contamination of drinking water and hands, respectively, and reduce reported diarrhea. ${ }^{1-4}$ However, hands are rapidly recontaminated by contact with objects and surfaces ${ }^{5-7}$ and treated water can be recontaminated by hands and utensils during storage. ${ }^{8}$ Sanitation interventions target reducing contamination further upstream by isolating fecal matter from the ambient surroundings but sanitation improvements to date have generally not reduced fecal indicators in the environment and shown mixed impact on health. ${ }^{9-12}$

Combining water, sanitation, and hygiene improvements could yield synergistic benefits. Coupling water treatment and

Received: September 12, 2018

Accepted: September 26, 2018

Published: September 26, 2018 


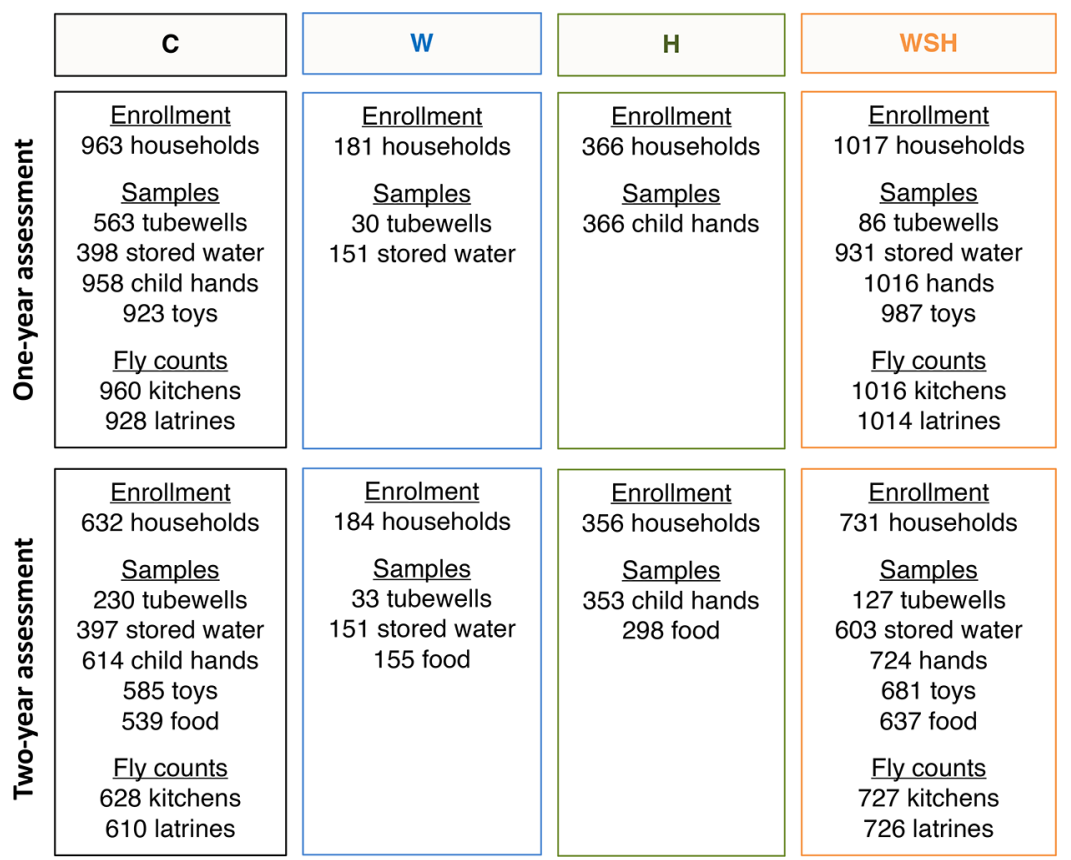

Figure 1. Flowchart of participant enrollment and environmental sampling scheme. C represents pooled data from (i) the control arm and (ii) the nutrition arm. WSH represents pooled data from (i) the combined water, sanitation and handwashing arm and (ii) the nutrition plus combined water, sanitation and handwashing arm. $\mathrm{W}$ refers to the individual water treatment arm, and $\mathrm{H}$ to the individual handwashing arm.

safe storage with a hygiene intervention could reduce recontamination of treated water through cleaner hands, whereas combining handwashing with sanitation improvements could reduce recontamination of washed hands through reduced exposure to fecal matter in the environment. Water, sanitation, and hygiene interventions could also work in concert to reduce food contamination by making treated water available for food preparation, facilitating handwashing before food handling and reducing breeding sites for flies transmitting pathogens to stored food. ${ }^{13,14}$ No studies have investigated whether combined water, sanitation, and hygiene interventions more effectively reduce contamination of drinking water, food, hands and fomites than individual interventions. We conducted an environmental assessment within a randomized controlled trial in Bangladesh (WASH Benefits, ClinicalTrials.gov NCT01590095) to assess whether (1) water, sanitation and hygiene interventions vs controls and (2) combined vs single water, sanitation, and hygiene interventions reduce fecal indicator bacteria and flies in the domestic environment.

\section{MATERIALS AND METHODS}

Study Design. WASH Benefits enrolled participants in four districts (Gazipur, Kishoreganj, Mymensingh, Tangail) in rural Bangladesh. These areas were selected because their groundwater chemistry was suitable for the trial's chlorinebased water treatment intervention ${ }^{15}$ and because they had no other major ongoing or planned water, sanitation, and hygiene programs. The trial enrolled pregnant women identified by screening the study area. Using global positioning system (GPS) coordinates, eight adjacent eligible women were grouped into clusters, and each eight clusters formed a block. Clusters were block-randomized into study arms by an off-site investigator (BFA), providing pair-matched randomization. WASH Benefits followed the birth cohort born to the enrolled pregnant women ("index children") for two years. Further details of the trial design have been previously described. ${ }^{16}$ The primary outcomes of WASH Benefits were child diarrhea and growth, and additional outcomes included protozoan and soil-transmitted helminth (STH) infections; these have been reported separately. ${ }^{17-19}$ Measures of environmental contamination were prespecified intermediate outcomes. $^{16}$

The trial had six intervention arms including single and combined water, sanitation, handwashing, and nutrition interventions and a double-sized control arm receiving no intervention. The intervention arms included (1) water treatment: point-of-use water treatment with sodium dichloroisocyanurate (NaDCC, Aquatabs) (Medentech, Wexford, Ireland) and safe storage in a narrow-mouth, lidded container with spigot, (2) sanitation improvements: upgrades to concrete-lined double-pit latrines, and provision of child potties and scoops for feces disposal, (3) handwashing promotion: provision of handwashing stations in the kitchen and latrine areas with a water reservoir, a bottle of soapy water mixture and a basin for rinsewater (4) nutrition improvements: provision of lipid-based nutrient supplements for children aged 6-24 months, recommendations for exclusive breastfeeding for children aged up to 6 months and age-appropriate nutrition recommendations from pregnancy through 24 months of age, (5) combined water, sanitation and handwashing (WSH), and (6) nutrition plus combined WSH $(\mathrm{N}+\mathrm{WSH})$.

Community health promoters hired from among local women and trained specifically for the study visited intervention households six times per month on average to demonstrate and encourage the targeted behaviors (e.g., water treatment, handwashing at critical times, latrine use for defecation) and supply intervention products for free throughout the trial period. Control households did not receive any interventions or health promoter visits. User adherence to the interventions was measured through spotcheck and structured observations in unannounced household visits (as an independent investigation from the study activities 
reported here). ${ }^{20}$ These demonstrated high adherence throughout the trial, including hardware availability observed by spot-checks and user practices assessed by structured observations. Spot-checks indicated $>95 \%$ of households in arms receiving the sanitation intervention had a latrine with a water seal compared to $23 \%$ of controls, and $>77 \%$ of households in arms receiving the handwashing intervention had water and soap present $<6$ steps from the kitchen or latrine compared to $21 \%$ of controls. ${ }^{20}$ Structured observations showed in $>94 \%$ of households in sanitation arms adults used a hygienic latrine for defecation (vs $40 \%$ of controls), in $>67 \%$ of households in handwashing arms participants washed hands with soap after using the latrine (vs 29\% of controls) and in $>65 \%$ of households in water treatment arms participants drank chlorine-treated water from a safe storage container (vs none of controls). ${ }^{20}$ Further details of user adherence have been described. ${ }^{20-22}$

Procedures. A subset of trial participants in the control, nutrition, WSH and $\mathrm{N}+\mathrm{WSH}$ arms was enrolled in an environmental enteric dysfunction (EED) substudy. EED enrollment was based on proximity to the laboratory and therefore (unlike the parent trial) not geographically pairmatched. We conducted an environmental assessment among EED substudy participants. This allowed us to assess the environmental impact of the combined WSH intervention by comparing pooled data from the WSH and $\mathrm{N}+\mathrm{WSH}$ arms (both receiving the WSH package, referred to as "WSH arm" hereinafter) to pooled data from the control and nutrition arms (neither one receiving the WSH intervention, referred to as "control arm" hereinafter). We also sampled a random subset of households in the single water and handwashing arms to assess the impact of combined vs individual interventions. The sanitation arm was not sampled as a previous assessment showed no early environmental impact in this $\operatorname{arm}^{23}$ and a separate longitudinal study is underway to assess the long-term effect of sanitation on environmental contamination (manuscript forthcoming). Sampling was conducted at two time points, approximately 1 and 2 years after intervention initiation, to match the timing of the trial's health outcome measurements (see Supporting Information (SI) Text S1 and Figure S1 for details of all environmental assessments nested within WASH Benefits).

Trained field workers from the International Centre for Diarrhoeal Disease Research, Bangladesh (icddr,b) visited households enrolled in the environmental assessment to collect samples. In the control and WSH arms, we quantified fecal indicator bacteria in drinking water, on index child hands, on toy ball "sentinel objects" and (in year two only) in food served to young children (Figure 1). Sentinel objects are presterilized objects (e.g., toy ball) left in the household to take up contamination from the domestic environment while household members interact with it and then tested for fecal indicator bacteria. ${ }^{24,25}$ They serve as a measure of overall contamination of domestic surfaces and objects, and have been shown to distinguish households with vs without improved water, sanitation, and hygiene conditions in Bangladesh. ${ }^{26,27}$ In single intervention arms, we only collected a relevant subset of sample types expected to be directly affected by the interventions; we sampled drinking water and (in year two) children's food in the water arm, and index child hands and (in year two) children's food in the handwashing arm. Additionally, field staff examined caregiver and index child hands (fingernails, fingerpads and palms) in the control, handwashing and WSH arms for visible dirt; observed dirt on hands has been validated as a proxy for handwashing ${ }^{28}$ and shown to correlate with bacterial counts on hands. ${ }^{29}$ Field staff also enumerated and speciated synanthropic flies in the kitchen and latrine areas in the control and WSH arms.

Sample Collection. Samples were collected using sterile Whirlpak bags (Nasco Modesto, Salida, CA). Clean gloves were worn while collecting hand rinse, toy rinse, and food samples. To collect drinking water, field workers asked the respondent to provide a glass of water in the same manner they would give it to their children and pour approximately $150 \mathrm{~mL}$ into the Whirlpak; field workers recorded if the water was provided from the source (tubewell) or from a storage container. If the respondent reported using chlorine, sodium thiosulfate was used to neutralize residual chlorine, and an additional sample was collected to measure the free chlorine residual with a digital colorimeter (Hach Pocket Colorimeter II). To sample child hands, field workers asked the respondent to place the index child's left-hand into a Whirlpak prefilled with $250 \mathrm{~mL}$ of distilled water. The hand was massaged from outside the bag for $15 \mathrm{~s}$ and shaken for $15 \mathrm{~s}$. The procedure was repeated with the right-hand in the same bag, and the rinsewater was preserved in Whirlpak. To sample sentinel objects, field workers delivered presterilized nonporous plastic toy balls to participants and instructed them to let the index child as well as other children in the compound play freely with the ball. They returned $24 \mathrm{~h}$ later to rinse the ball in a Whirlpak prefilled with $250 \mathrm{~mL}$ of distilled water by massaging it from outside the bag and shaking the bag, for $15 \mathrm{~s}$ each. The rinsewater was preserved for analysis and the toy was left at the household; a new ball was delivered at the time of the year two sampling. To sample food, field workers identified stored food prepared to be served to children $<5$ years and asked the respondent to provide a small amount in the same manner they would serve it to their children. They prioritized sampling rice if available. Food was scooped from the dish that it was provided in into a $50 \mathrm{~mL}$ sterile tube using a sterile spoon. Samples were transported to the icddr,b field laboratory on ice at $2-8{ }^{\circ} \mathrm{C}$ and analyzed within $12 \mathrm{~h}$ of collection.

Sample Processing. We used membrane filtration to analyze water samples (undiluted) and hand and toy rinse samples (both undiluted and with 1:10 and 1:100 dilutions). $100 \mathrm{~mL}$ aliquots were filtered through a $0.45-\mu \mathrm{m}$ cellulose filter and incubated for $24 \mathrm{~h}$ on $60 \mathrm{~mm}$ MI agar plates (BD Difco, Franklin Lakes, NJ). Water and hand rinse samples were incubated at $35{ }^{\circ} \mathrm{C}$ to enumerate Escherichia coli and total coliforms following standard protocols. ${ }^{30} \mathrm{We}$ modified the protocol to incubate toy rinse samples at $44.5{ }^{\circ} \mathrm{C}$ to enumerate E. coli and fecal/thermotolerant coliforms ${ }^{31}$ as a previous study found that fecal (rather than total) coliforms on toy balls were a more sensitive indicator of environmental contamination. ${ }^{26}$

We used the pour-plate technique to analyze food samples. $10 \mathrm{~g}$ food aliquots were homogenized with $40 \mathrm{~mL}$ of distilled water. The homogenate was analyzed both undiluted and with 1:100 dilution. A $2.5 \mathrm{~mL}$ aliquot of homogenate and $15 \mathrm{~mL}$ of TBX media (Sigma-Aldrich, St. Louis, MO) were added to a $100 \mathrm{~mm}$ Petri dish by pour-plate and incubated for $24 \mathrm{~h}$ at 44.5 ${ }^{\circ} \mathrm{C}$ to enumerate E. coli following standard protocols. ${ }^{32}$ An additional $5 \mathrm{~g}$ food aliquot was oven-dried overnight to determine moisture content.

Counts were expressed in colony forming units (CFU) (per $100 \mathrm{~mL}$ for water samples, per 2 hands for child hands, per 1 toy for sentinel toys and per 1 dry gram for food). Plates with 

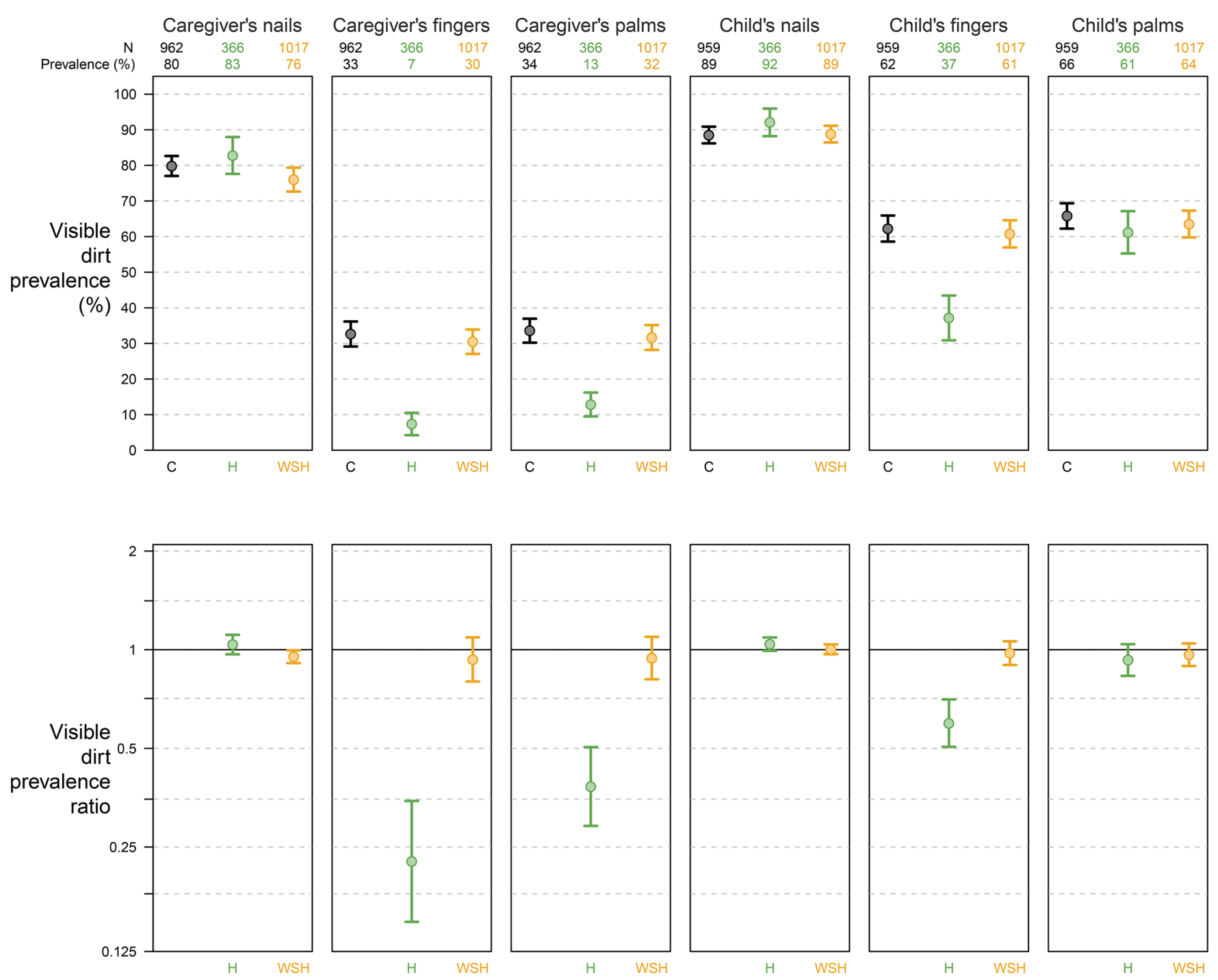

Figure 2. Prevalence of caregivers and children with visible dirt on hands at year one. C represents pooled data from (i) the control arm and (ii) the nutrition arm. WSH represents pooled data from (i) the combined water, sanitation and handwashing arm and (ii) the nutrition plus combined water, sanitation and handwashing arm. $\mathrm{H}$ refers to the individual handwashing arm.

$>200$ colonies for water, hand rinses and toy rinses and $>500$ colonies for food were classified as too numerous to count (TNTC). The higher detection limit for the food samples reflects the larger surface area of the $100 \mathrm{~mm}$ plates, allowing a higher number of colonies to be visually distinguished (see SI Table S1 for detection limits for each sample type).

Quality Control. One laboratory control per analyst per day and 5\% replicates (repeat aliquots from same Whirlpak for every 20th sample) were processed. Field workers collected $10 \%$ field blanks (one blank for every 10 samples) by asking respondents to pour distilled water from a sterile bottle into a Whirlpak as if collecting a water sample, by opening and massaging a prefilled Whirlpak as if sampling a hand, and by rinsing a presterilized toy ball in prefilled Whirlpak as if sampling a toy.

Fly Counts. To enumerate flies, field workers identified a suitable location in the kitchen and latrine areas (away from the stove smoke, under a roof or protected from rain) and horizontally hung three 1.5 -foot strips of nonbaited sticky fly tape. They returned to the household $24 \mathrm{~h}$ later to count the captured synanthropic flies and speciate them according to a visual identification chart. ${ }^{33}$
Ethics. Participants provided written informed consent in the local language (Bengali). The study protocol was approved by human subjects committees at the icddr,b (PR-11063), University of California, Berkeley (2011-09-3652), and Stanford University (25863).

Statistical Methods. We prespecified and registered our analysis plan on Open Science Framework (https://osf.io/ $6 \mathrm{u} 7 \mathrm{cn} /)$.

Sample Size. The EED substudy, within which the environmental sampling was nested, targeted 1500 households (375/arm) and enrolled approximately 2000 households in year one to allow for attrition by year two. In addition, we enrolled 180 water arm households and 360 handwashing arm households in the environmental assessment. We obtained measures of contamination and intraclass correlation coefficients from the literature and unpublished pilot data (see analysis plan). We used a one-sided $\alpha$ of 0.05 , assuming that the interventions would decrease but not increase contamination. ${ }^{1,2}$ Our sample size yielded $80 \%$ power to detect a 0.20 $\log _{10}$ reduction in E. coli concentration in drinking water, hand rinses and toy rinses at each sampling time point, compared to controls. 

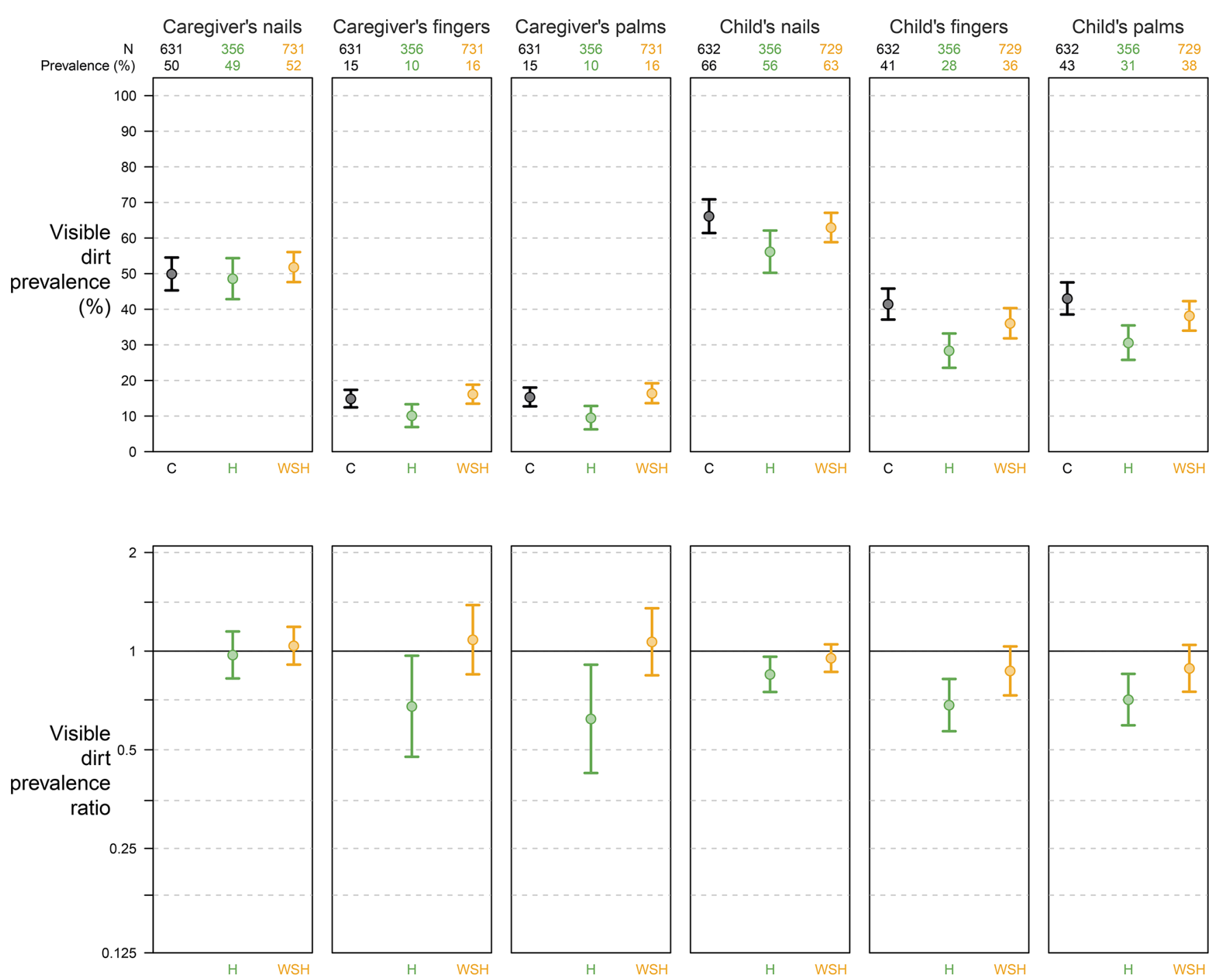

Figure 3. Prevalence of caregivers and children with visible dirt on hands at year two. C presents pooled data from (i) the control arm and (ii) the nutrition arm. WSH represents pooled data from (i) the combined water, sanitation and handwashing arm and (ii) the nutrition plus combined water, sanitation and handwashing arm. $\mathrm{H}$ refers to the individual handwashing arm.

Parameters of Interest. Our outcomes were (1) prevalence and concentration of E. coli and total/fecal coliforms, (2) prevalence and number of flies near the kitchen and latrine, and (3) prevalence of caregiver and child hands with visible dirt. Our parameters were prevalence ratios (PR) and differences (PD) for binary measures, $\log _{10}$ reductions for $E$. coli and coliform concentrations, and fly count ratios for the number of flies. We substituted bacterial counts with half the lower detection limit for nondetects, $200 \mathrm{CFU}$ for TNTC water, hand rinse and toy rinse samples and $500 \mathrm{CFU}$ for TNTC food samples. ${ }^{34}$ Means for multiple dilutions were obtained by dividing the sum of plate counts by the total sample volume filtered for all countable plates.

Estimation Strategy. We compared all intervention arms to controls and the combined WSH arm to the single water and handwashing arms. Analyses were intention-to-treat; this preserves the randomization and is appropriate given the high intervention adherence. ${ }^{20}$ Randomization balanced covariates across arms. ${ }^{17}$ Therefore, we relied on unadjusted estimates in our analysis. We estimated unadjusted parameters using generalized linear models with robust standard errors, and a log link for PRs, linear link for PDs and $\log _{10}$ reductions, and log link allowing for overdispersion (negative binomial regression) for fly count ratios. Secondary analyses adjusted for prespecified covariates using doubly robust targeted maximum likelihood estimation (TMLE) incorporating an ensemble machine learning method called Super Learner. ${ }^{35,36}$ We conducted separate comparisons at each of the two measurement rounds and performed a Bonferroni correction for multiple measurements by multiplying the p-values for effect estimates by two. We conducted separate analyses stratifying by whether the sampled drinking water came from the source or storage container. We assessed effect modification by season by including an interaction term for wet vs dry season; Bangladesh has a monsoon season (June-October), during which it receives $>80 \%$ of its rain $^{37}$ and environmental contamination levels typically increase. $^{38}$

\section{RESULTS AND DISCUSSION}

Enrollment. Of 2445 households selected for EED enrollment in year one, we enrolled 1980 (81\%) in the environmental assessment between October 2013 and December 2014; we also enrolled 181 water arm households 

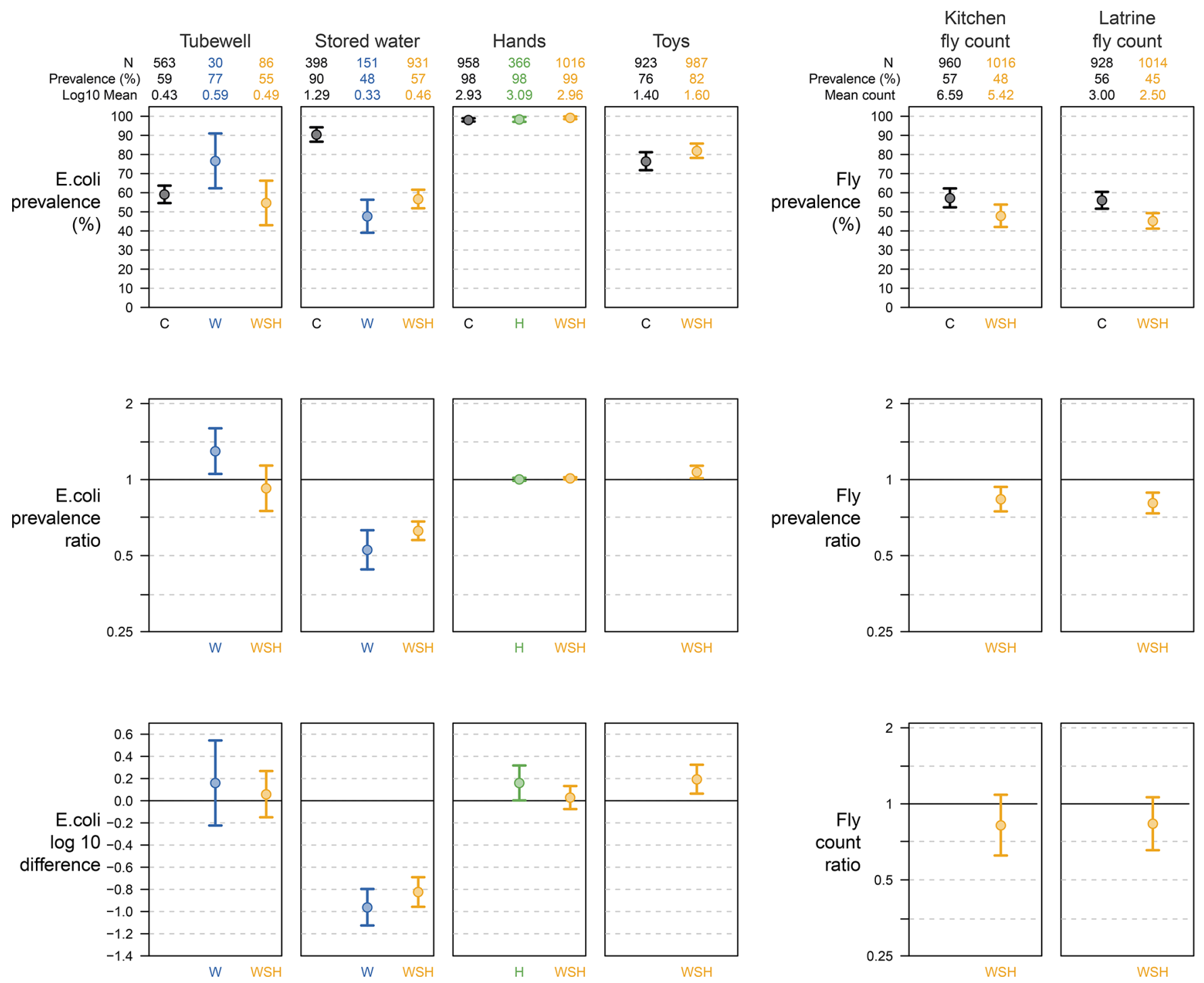

Figure 4. Prevalence and concentration of E. coli (in source and stored drinking water, child hand rinses, sentinel toy rinses) and prevalence and number of flies captured near kitchen and latrine at year one. E. coli concentrations are reported in log colony forming units (CFU) per $100 \mathrm{~mL}$ for drinking water samples (tubewell and stored water), per two hands for child hand rinses and per toy for sentinel toy rinse samples. C represents pooled data from (i) the control arm and (ii) the nutrition arm. WSH represents pooled data from (i) the combined water, sanitation and handwashing arm and (ii) the nutrition plus combined water, sanitation and handwashing arm. W refers to the individual water treatment arm, and $\mathrm{H}$ to the individual handwashing arm.

and 366 handwashing arm households. At year two, among 1515 households successfully enrolled in the EED substudy, we enrolled 1363 (90\%) in the environmental assessment between May 2015 and May 2016; we also enrolled 184 water arm households and 356 handwashing arm households. Reasons for households not being enrolled were no live birth or index child death ( $10 \%$ in year one, $0.3 \%$ in year two), absence or relocation ( $7 \%$ in year one, $4 \%$ in year two) and refusal (2\% in year one, $5 \%$ in year two). Covariates were balanced between arms among enrolled households (SI Table S2).

Observed Hand Cleanliness. Among caregivers in the control arm, dirt was observed on $80 \%$ of nails and $33-34 \%$ of fingerpads and palms at year one, and $50 \%$ of nails and $15 \%$ of fingerpads and palms at year two (Figures 2 and 3). Among children in the control arm, dirt was observed on $89 \%$ of nails and $62-66 \%$ of fingerpads and palms at year one (mean child age: 14 months), and $66 \%$ of nails and $41-43 \%$ of fingerpads and palms at year two (mean child age: 30 months); these ages roughly coincided with $\mathrm{WHO}$ windows for rolling, crawling and learning to walk (5-18 months) vs walking well (>18 months). ${ }^{39}$ Caregivers in the handwashing arm were substantially less likely to have dirt on fingerpads and palms but not under fingernails at both time points (Figures 2 and 3 ). Similarly, children in the handwashing arm were less likely to have dirt on fingerpads and palms and, to a smaller extent, under fingernails (Figures 2 and 3). Caregivers or children in the WSH arm were no less likely to have dirt on their hands than controls at either time point (Figures 2 and 3 ).

Fecal Contamination. Levels of contamination. We analyzed 6409 samples at year one and 6127 at year two. Of drinking water samples, $29 \%$ were provided directly from a tubewell and $71 \%$ from a storage container (in arms receiving the water intervention, this was predominantly the provided safe storage container). Among controls, we detected E. coli in 59-62\% of tubewells, $90-93 \%$ of stored drinking water, 96- 

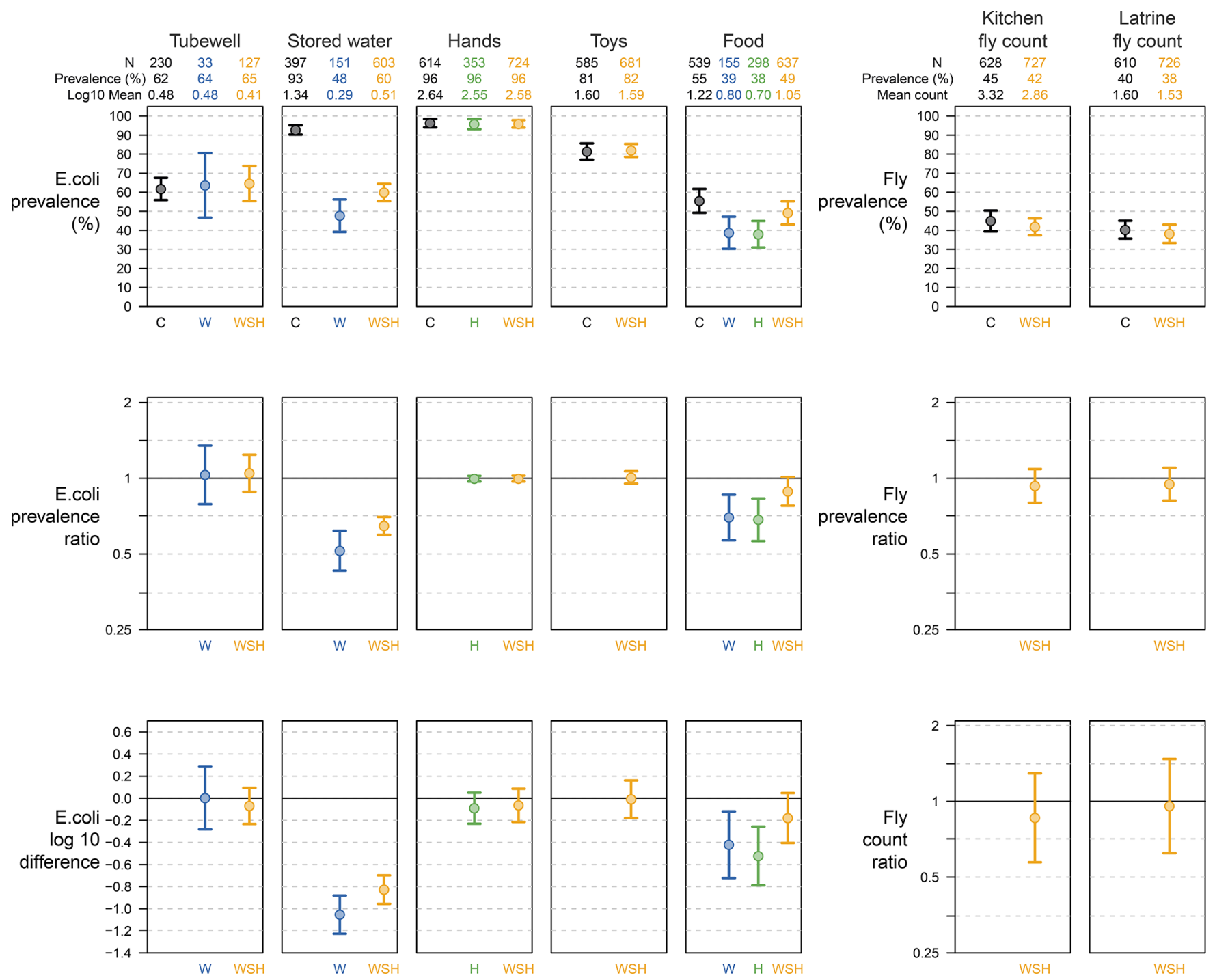

Figure 5. Prevalence and concentration of E. coli (in source and stored drinking water, child hand rinses, sentinel toy rinses, and food given to young children) and prevalence and number of flies captured near kitchen and latrine at year two. E. coli concentrations are reported in log colony forming units (CFU) per $100 \mathrm{~mL}$ for drinking water samples (tubewell and stored water), per two hands for child hand rinses, per toy for sentinel toy rinse samples and per dry gram for food samples. C represents pooled data from (i) the control arm and (ii) the nutrition arm. WSH represents pooled data from (i) the combined water, sanitation and handwashing arm, and (ii) the nutrition plus combined water, sanitation and handwashing arm. $\mathrm{W}$ refers to the individual water treatment arm, and $\mathrm{H}$ to the individual handwashing arm.

$98 \%$ of child hands and $76-81 \%$ of toys at the two time points and $55 \%$ of children's food at year two (Figures 4 and 5 ).

Intervention Effects. Among households receiving water treatment interventions, $90 \%$ of stored drinking water samples were reported to be chlorinated, and of these, $80 \%$ had detectable $(>0.1 \mathrm{mg} / \mathrm{L})$ free chlorine residual at the two time points, while $1 \%$ of controls reported water treatment. Compared to controls, stored drinking water in the single water arm had approximately $50 \%$ reduction in E. coli prevalence and 1-log reduction in $E$. coli concentration at both time points (Yr1: prevalence ratio $[\mathrm{PR}]=0.53(0.44$, $0.63), \Delta \log _{10}=-0.96(-1.13,-0.80) ;$ Yr2: PR $=0.51(0.43$, $0.62), \Delta \log _{10}=-1.05(-1.23,-0.88)$; all Bonferronicorrected $p<0.001$ ) (Figures 4 and 5, SI Tables S3 and S4). Compared to controls, stored drinking water in the WSH arm also had similar reductions in $E$. coli prevalence and concentration at both time points $(\mathrm{Yr} 1: \mathrm{PR}=0.63(0.58,0.68)$, $\Delta \log _{10}=-0.82(-0.96,-0.69) ; \mathrm{Yr} 2: \mathrm{PR}=0.65(0.59,0.70)$, $\Delta \log _{10}=-0.83(-0.96,-0.70)$; all Bonferroni-corrected $p<$
0.001) (Figures 4 and 5, SI Tables S3 and S4). Compared to controls, food (measured only in year two) had approximately $30 \%$ reduction in $E$. coli prevalence in the water arm $(\mathrm{PR}=$ $0.70(0.57,0.86))$ and handwashing arm $(\mathrm{PR}=0.68(0.56$, $0.83)$ ) and a borderline reduction in the WSH arm $(\mathrm{PR}=0.89$ $(0.78,1.01))$. Similarly, food $E$. coli counts were reduced by about $0.5-\log$ in the water $\operatorname{arm}\left(\Delta \log _{10}=-0.42(-0.72\right.$, $-0.12))$ and handwashing arm $\left(\Delta \log _{10}=-0.52(-0.79\right.$, $-0.26))$ and borderline reduced in the WSH arm $\left(\Delta \log _{10}=\right.$ $-0.18(-0.40,0.05)$ ) compared to controls (Figure 5, SI Table S4). Tubewell water, child hands and sentinel toys had no reductions in $E$. coli prevalence or concentration in intervention arms vs controls at either time point (Figures 4 and 5, SI Tables S3 and S4). Compared to controls, E. coli counts were borderline increased on child hands in the handwashing arm and on toys in the WSH arm at year one but not at year two (Figures 4 and 5, SI Tables S3 and S4).

Comparing combined vs individual interventions, the WSH arm had higher stored water E. coli prevalence and 
concentration than the water arm at year two $(\mathrm{PR}=1.26$ $(1.05,1.50), \Delta \log _{10}=0.23(0.09,0.36)$, both Bonferronicorrected $p<0.05)$; there was a similar albeit borderline nonsignificant pattern at year one (SI Tables S3 and S4). The WSH arm also had higher food E. coli prevalence and concentration than the water arm $(\mathrm{PR}=1.27(1.02,1.57)$, $\left.\Delta \log _{10}=0.24(-0.03,0.52)\right)$ and handwashing arm $(\mathrm{PR}=1.30$ $\left.(1.08,1.55), \Delta \log _{10}=0.34(0.12,0.57)\right)($ SI Tables S3 and S4). There were no differences in E. coli prevalence or concentration in the other sample types between combined and individual intervention arms.

Secondary analyses adjusting for confounders yielded similar results except that food E. coli reductions were slightly attenuated (SI Tables S3 and S4). Subgroup analyses suggested overall lower contamination and larger reductions in E. coli in stored drinking water and food from the interventions during the dry season; there were no other seasonal effects (SI Tables S5 and S6). Total/fecal coliforms showed patterns similar to E. coli (SI Tables S7 and S8).

Quality Control. Across sample types and sampling rounds, $23 \%$ of samples were nondetect and $6 \%$ exceeded upper detection limits. Intraclass correlation between replicates was $>85 \%$ at both time points. E. coli was detected in $2.5 \%$ of blanks and the geometric mean E. coli count among positive blanks was 7 CFU. Repeating the analyses after removing the data from days with contaminated blanks did not change findings (SI Tables S9 and S10).

Fly Presence. At year one, at least 1 fly was captured near the kitchen in $57 \%$ and near the latrine in $56 \%$ of control households; the mean number of flies was 6.59 (range: 1-201) near the kitchen and 3.00 (range: 1-229) near the latrine. At year two, at least 1 fly was captured near the kitchen in $45 \%$ and near the latrine in $40 \%$ of control households; the mean number of flies was 3.32 (range: 1-86) near the kitchen and 1.60 (range: 1-98) near the latrine. The predominant fly species was the common housefly (Musca domestica). Compared to controls, fly prevalence was reduced significantly in the WSH arm at year one, both near the kitchen $(\mathrm{PR}=0.83$ $(0.75,0.94)$, Bonferroni-corrected $p<0.005)$ and the latrine $(\mathrm{PR}=0.81(0.74,0.89)$, Bonferroni-corrected $p<0.001)$, but not at year two (Figures 4 and 5). There was no consistent impact on fly numbers at either time point (Figures 4 and 5).

Implications. Water, sanitation, and handwashing interventions reduced fecal exposure along the direct transmission pathways of stored drinking water and food but not along the indirect, more upstream pathways of child hands, household objects and groundwater sources. Chlorine and safe storage effectively reduced $E$. coli in stored water, consistent with prior evidence.

E. coli in food was reduced in the water and handwashing arms. This could indicate that participants used treated water to prepare food and rinse utensils in the water arm and improved their handwashing around food handling in the handwashing arm. In structured observations, $5 \%$ of caregivers in the handwashing arm washed hands specifically before preparing food vs $0.5 \%$ of controls, suggesting hand hygiene around food handling remained poor. ${ }^{20}$ However, overall handwashing practices improved; in structured observations, $67 \%$ of caregivers in the handwashing arm washed hands after using the latrine vs $29 \%$ of controls. ${ }^{20}$ This supports our observation that caregivers in the handwashing arm were significantly less likely to have visible dirt on fingerpads and palms (but not nails) than controls. The hand observation method has the caveat that field staff could overperceive hand cleanliness in the handwashing arm as they were not blinded to the intervention assignment ${ }^{40}$ (blinding was not feasible since the interventions had distinct hardware components). However, the staff perceived no such effect in the WSH arm, suggesting no blanket bias from knowledge of treatment status.

Similarly, children in the handwashing arm had less dirt on fingerpads and palms, and slightly less dirt under nails. As the children were too young to wash their own hands, this might indicate that improved hygiene among caregivers translated into washing or wiping child hands. However, the interventions did not reduce fecal bacteria on child hands. This could be because our sampling method of massaging and vigorously rinsing hands might have eluted dirt from under fingernails that was not removed by washing/wiping. Our previous work found that young children in our study population frequently touch soil, which is highly contaminated in this setting. ${ }^{38,41}$ Soil trapped under nails might have harbored sufficient E. coli to undermine the effect of cleaner fingers and palms; fingernails have been shown to harbor pathogens such as parasite eggs and larvae in Bangladesh. ${ }^{42}$

The interventions did not reduce overall contamination in the ambient domestic environment, as measured by sentinel toys and groundwater samples. This is consistent with an earlier assessment among study households that found no difference in fecal contamination in the ambient environment (courtyard soil, ponds, groundwater) in the sanitation and combined WSH arms after approximately 4 months of intervention. ${ }^{23}$ Our findings differ from two studies in rural Bangladesh that found fewer fecal coliforms on sentinel toys in compounds with better water, sanitation and hygiene infrastructure. $^{26,27}$ However, these studies used cross-sectional designs susceptible to confounding; compounds with improved sanitary conditions could have other characteristics leading to reduced contamination. Toy balls in our study were often coated in soil; the lack of $E$. coli reduction on toys is consistent with the lack of intervention impact on $E$. coli in soil in our previous assessment. $^{23}$

Our findings also suggest that single interventions achieved larger reductions in contamination than the combined WSH package. The water arm had less E. coli in stored water than the WSH arm, and the water and handwashing arms had less E. coli in food than the WSH arm. Similarly, children and caregivers in the handwashing but not the WSH arm were less likely to have visible dirt on hands. One explanation could be that the multicomponent package diffused the effectiveness of any one intervention; that is, targeting a specific behavior was more effective than attempting to change multiple behaviors. ${ }^{43}$ However, spot-checks and structured observations revealed only minor differences in uptake indicators between combined and single intervention arms. ${ }^{20}$

Environmental Findings in the Context of the Trial'S Health Outcomes. WASH Benefits measured enteric infections in children including (1) caregiver-reported diarrhea, (2) protozoan infections with Cryptosporidium spp., Giardia duodenalis, E. histolytica measured by qPCR of fecal specimens, and (3) STH infections with Ascaris lumbricoides, Trichuris trichiura, and hookworm measured by Kato-Katz analysis of fecal specimens. These measurements showed reduced diarrhea in all arms except for the single water arm ${ }^{17}$ and reduced Giardia infection in all arms except for the single water and nutrition arms; ${ }^{18}$ Cryptosporidium and E. histolytica were too rare to assess impact. Hookworm was reduced in the 
single water and sanitation arms as well as the combined WSH arm. ${ }^{19}$ The discrepancies between intervention impacts on $E$. coli vs health outcomes point to the complexity of environmentally mediated pathogen transmission, likely resulting from the diversity of fecal-oral pathogens, temporal fluctuations in transmission dynamics, ${ }^{44,45}$ differences in environmental survival, $^{46-48}$ varying resistance to disinfection ${ }^{49,50}$ and imperfect correlations between fecal indicator bacteria and pathogens. $^{51}$

Our E. coli measurements demonstrate a clear reduction in fecal exposure through stored drinking water in water intervention arms; this finding is consistent with our finding of reduced hookworm infection in the single water arm. ${ }^{19}$ The lack of diarrhea reduction in this arm may indicate that water was not a dominant transmission pathway for diarrheagenic pathogens in this population during this study. A previous study in a similar rural Bangladeshi population found $36 \%$ diarrhea reduction from the same water intervention, ${ }^{52}$ suggesting larger effect at a time of potentially more pronounced waterborne transmission. Another explanation is that measured reductions in chlorine-sensitive indicator bacteria do not indicate reductions in chlorine-resistant pathogens. Some viruses can withstand chlorine, ${ }^{49}$ and protozoan cysts are highly chlorine-resistant. ${ }^{50}$ If dominant diarrheagenic pathogens in the study population during the trial were chlorine-resistant, E. coli reductions in water would not translate to reductions in diarrhea. This is consistent with our finding that the single water arm had no reduction in infections with chlorine-resistant Giardia. ${ }^{18}$ Cryptosporidium, another chlorine-resistant protozoan pathogen, ${ }^{50}$ is a major cause of diarrhea in Bangladesh. ${ }^{53,54}$ Cryptosporidium infections (measured at the year two time point) were rare in our study; ${ }^{18}$ settings with high Cryptosporidium incidence would be another example where chlorination may reduce indicator bacteria in drinking water but not affect diarrhea. Our E. coli measurements also demonstrate a reduction in fecal exposure through food in the handwashing arm and, coupled with our observation of less dirt on caregiver hands in this arm, suggest reduced caregiver hand contamination. This is consistent with the reductions in diarrhea and Giardia infection in the handwashing arm. ${ }^{17,18}$ In contrast, STH infections were not reduced in this arm. ${ }^{19}$ This could be because the handwashing intervention did not remove dirt from fingernails; STH ova and larvae are detected under fingernails ${ }^{42}$ and nail clipping reduces parasite infection. ${ }^{55}$ Finally, the E. coli reductions in stored water and food in the combined WSH arm support the reductions in diarrheal, protozoan and $\mathrm{STH}$ infections in this arm, and the lack of synergistic impact on fecal contamination from combining WSH interventions is consistent with the lack of incremental health benefits in this arm. ${ }^{17-19}$

Our findings confirm that the use of E. coli as an indicator of water treatment effectiveness for chlorine-sensitive pathogens is appropriate but the alignment between E. coli measurements and infectious disease end points is heterogeneous and pathogen-specific. Our findings indicate that water, sanitation, and hygiene improvements reduced fecal contamination along the direct transmission pathways of stored drinking water and food but not in the overall ambient environment in this setting. These findings support implementing water treatment and handwashing to reduce fecal exposures through drinking water and food in low-income countries but provide no evidence that combining interventions further reduces exposure.

\section{ASSOCIATED CONTENT}

\section{Supporting Information}

The Supporting Information is available free of charge on the ACS Publications website at DOI: 10.1021/acs.est.8b05153.

Text S1. Environmental assessments nested within WASH Benefits Figure S1. Types of samples collected for environmental assessments approximately 4 months, one year and two years after the initiation of WASH Benefits interventions Table S1. E. coli detection units and limits Table S2. Enrollment characteristics by intervention group Table S3. E. coli prevalence and concentration measured in control, water treatment, handwashing and WSH arms after one year of intervention Table S4. E. coli prevalence and concentration measured in control, water treatment, handwashing and WSH arms after two years of intervention Table S5. Subgroup analysis by dry vs wet season on E. coli prevalence at year one Table S6. Subgroup analysis by dry vs wet season on E. coli prevalence at year two Table S7. Total/fecal coliform prevalence and concentration measured in control, water treatment, handwashing and WSH arms after one year of intervention Table S8. Total/fecal coliform prevalence and concentration measured in control, water treatment, handwashing and WSH arms after two years of intervention Table S9. E. coli prevalence and concentration at year one (after data from dates with contaminated blanks have been removed) Table S10. E. coli prevalence and concentration at year two (after data from dates with contaminated blanks have been removed) (PDF)

\section{AUTHOR INFORMATION}

\section{Corresponding Author}

*E-mail: aercume@ncsu.edu. ORCID $\odot$

Ayse Ercumen: 0000-0001-6002-1514

Notes

The authors declare no competing financial interest.

\section{ACKNOWLEDGMENTS}

This research protocol was funded by Global Development grant OPPGD759 from Bill \& Melinda Gates Foundation to University of California, Berkeley to International Center for Diarrhoeal Disease Research, Bangladesh (icddr,b, grant number 741). icddr,b acknowledges with gratitude the commitment of Bill \& Melinda Gates Foundation and University of California, Berkeley to its research efforts. icddr,b is also grateful to the Governments of Bangladesh, Canada, Sweden and the UK for providing core/unrestricted support. We also offer our sincere gratitude to the study participants who participated in the trial and community health promoters (CHPs), field workers and supervisors who delivered the interventions.

\section{REFERENCES}

(1) Arnold, B. F.; Colford, J. M. Treating Water with Chlorine at Point-of-Use to Improve Water Quality and Reduce Child Diarrhea in Developing Countries: A Systematic Review and Meta-Analysis. Am. J. Trop. Med. Hyg. 2007, 76 (2), 354-364.

(2) Luby, S. P.; Agboatwalla, M.; Raza, A.; Sobel, J.; Mintz, E. D.; Baier, K.; Hoekstra, R. M.; Rahbar, M. H.; Hassan, R.; Qureshi, S. M.; Gangarosa, E. J. Microbiologic Effectiveness of Hand Washing with 
Soap in an Urban Squatter Settlement, Karachi, Pakistan. Epidemiol. Infect. 2001, 127 (2), 237-244.

(3) Clasen, T. F.; Alexander, K. T.; Sinclair, D.; Boisson, S.; Peletz, R.; Chang, H. H.; Majorin, F.; Cairncross, S. Interventions to Improve Water Quality for Preventing Diarrhoea. In Cochrane Database of Systematic Reviews; John Wiley \& Sons, Ltd, 2015.

(4) Freeman, M. C.; Stocks, M. E.; Cumming, O.; Jeandron, A.; Higgins, J. P. T.; Wolf, J.; Prüss-Ustün, A.; Bonjour, S.; Hunter, P. R.; Fewtrell, L.; Curtis, V. Systematic Review: Hygiene and Health: Systematic Review of Handwashing Practices Worldwide and Update of Health Effects. Trop. Med. Int. Health 2014, 19 (8), 906-916.

(5) Sobel, J.; Mahon, B.; Mendoza, C. E.; Passaro, D.; Cano, F.; Baier, K.; Racioppi, F.; Hutwagner, L.; Mintz, E. Reduction of Fecal Contamination of Street-Vended Beverages in Guatemala by a Simple System for Water Purification and Storage, Handwashing, and Beverage Storage. Am. J. Trop. Med. Hyg. 1998, 59 (3), 380-387.

(6) Ram, P. K.; Jahid, I.; Halder, A. K.; Nygren, B.; Islam, M. S.; Granger, S. P.; Molyneaux, J. W.; Luby, S. P. Variability in Hand Contamination Based on Serial Measurements: Implications for Assessment of Hand-Cleansing Behavior and Disease Risk. Am. J. Trop. Med. Hyg. 2011, 84 (4), 510-516.

(7) Pickering, A. J.; Julian, T. R.; Mamuya, S.; Boehm, A. B.; Davis, J. Bacterial Hand Contamination among Tanzanian Mothers Varies Temporally and Following Household Activities. Trop. Med. Int. Health 2011, 16 (2), 233-239.

(8) Wright, J.; Gundry, S.; Conroy, R. Household Drinking Water in Developing Countries: A Systematic Review of Microbiological Contamination between Source and Point-of-Use. Trop. Med. Int. Health 2004, 9 (1), 106-117.

(9) Patil, S. R.; Arnold, B. F.; Salvatore, A. L.; Briceno, B.; Ganguly, S.; Jr, J. M. C.; Gertler, P. J. The Effect of India's Total Sanitation Campaign on Defecation Behaviors and Child Health in Rural Madhya Pradesh: A Cluster Randomized Controlled Trial. PLOS Med. 2014, 11 (8), e1001709.

(10) Clasen, T.; Boisson, S.; Routray, P.; Torondel, B.; Bell, M.; Cumming, O.; Ensink, J.; Freeman, M.; Jenkins, M.; Odagiri, M.; Ray, S.; Sinha, A.; Suar, M.; Schmidt, W. P. Effectiveness of a Rural Sanitation Programme on Diarrhoea, Soil-Transmitted Helminth Infection, and Child Malnutrition in Odisha, India: A ClusterRandomised Trial. Lancet Glob. Health 2014, 2 (11), e645-e653.

(11) Pickering, A. J.; Djebbari, H.; Lopez, C.; Coulibaly, M.; Alzua, M. L. Effect of a Community-Led Sanitation Intervention on Child Diarrhoea and Child Growth in Rural Mali: A Cluster-Randomised Controlled Trial. Lancet Glob. Health 2015, 3 (11), e701-e711.

(12) Sclar, G. D.; Penakalapati, G.; Amato, H. K.; Garn, J. V.; Alexander, K.; Freeman, M. C.; Boisson, S.; Medlicott, K. O.; Clasen, T. Assessing the Impact of Sanitation on Indicators of Fecal Exposure along Principal Transmission Pathways: A Systematic Review. Int. J. Hyg. Environ. Health 2016, 219 (8), 709-723.

(13) Doza, S.; Rahman, M. J.; Islam, M. A.; Kwong, L. H.; Unicomb, L.; Ercumen, A.; Pickering, A. J.; Parvez, S. M.; Naser, A. M.; Ashraf, S.; Das, K. K.; Luby, S. P. Prevalence and Association of Escherichia Coli and Diarrheagenic Escherichia Coli in Stored Foods for Young Children and Flies Caught in the Same Households in Rural Bangladesh. Am. J. Trop. Med. Hyg. 2018, tpmd170408.981031

(14) Parvez, S. M.; Kwong, L.; Rahman, M. J.; Ercumen, A.; Pickering, A. J.; Ghosh, P. K.; Rahman, M. Z.; Das, K. K.; Luby, S. P.; Unicomb, L. Escherichia Coli Contamination of Child Complementary Foods and Association with Domestic Hygiene in Rural Bangladesh. Trop. Med. Int. Health 2017, 22 (5), 547-557.

(15) Naser, A. M.; Higgins, E. M.; Arman, S.; Ercumen, A.; Ashraf, S.; Das, K. K.; Rahman, M.; Luby, S. P.; Unicomb, L. Effect of Groundwater Iron on Residual Chlorine in Water Treated with Sodium Dichloroisocyanurate Tablets in Rural Bangladesh. Am. J. Trop. Med. Hyg. 2018, tpmd160954.98977

(16) Arnold, B. F.; Null, C.; Luby, S. P.; Unicomb, L.; Stewart, C. P.; Dewey, K. G.; Ahmed, T.; Ashraf, S.; Christensen, G.; Clasen, T.; Dentz, H. N.; Fernald, L. C. H.; Haque, R.; Hubbard, A. E.; Kariger, P.; Leontsini, E.; Lin, A.; Njenga, S. M.; Pickering, A. J.; Ram, P. K.;
Tofail, F.; Winch, P. J.; Colford, J. M. Cluster-Randomised Controlled Trials of Individual and Combined Water, Sanitation, Hygiene and Nutritional Interventions in Rural Bangladesh and Kenya: The WASH Benefits Study Design and Rationale. BMJ. Open 2013, 3 (8), e003476.

(17) Luby, S. P.; Rahman, M.; Arnold, B. F.; Unicomb, L.; Ashraf, S.; Winch, P. J.; Stewart, C. P.; Begum, F.; Hussain, F.; Benjamin-Chung, J.; Leontsini, E.; Naser, A. M.; Parvez, S. M.; Hubbard, A. E.; Lin, A.; Nizame, F. A.; Kaniz, J.; Ercumen, A.; Ram, P. K.; Das, K. K.; Abedin, J.; Clasen, T. F.; Dewey, K. G.; Fernald, L. C.; Clair, N.; Tahmeed, A.; Colford, J. M. Effects of Water Quality, Sanitation, Handwashing, and Nutritional Interventions on Diarrhoea and Child Growth in Rural Bangladesh: A Cluster Randomised Controlled Trial. Lancet Glob. Health 2018, 6 (3), e302-e315.

(18) Lin, A.; Ercumen, A.; Benjamin-Chung, J.; Arnold, B. F.; Das, S.; Haque, R.; Ashraf, S.; Parvez, S. M.; Unicomb, L.; Rahman, M.; Hubbard, A. E.; Stewart, C. P.; Colford, J. M.; Luby, S. P. Effects of Water, Sanitation, Handwashing, and Nutritional Interventions on Child Enteric Protozoan Infections in Rural Bangladesh: A ClusterRandomized Controlled Trial. Clin. Infect. Dis. 2018. DOI: 10.1093/ $\mathrm{cid} /$ ciy320

(19) Ercumen, A.; Benjamin-Chung, J.; Arnold, B.; Lin, A.; Hubbard, A.; Stewart, C.; Parvez, S.; Unicomb, L.; Rahman, M.; Haque, R.; Colford, J. M.; Luby, S. P. Effects of Water, Sanitation, Handwashing and Nutritional Interventions on Soil Transmitted Helminth Infections in Young Children: A Cluster-Randomised Controlled Trial in Rural Bangladesh. 2018, (in preparation).

(20) Parvez, S. M.; Azad, R.; Rahman, M.; Unicomb, L.; Ram, P. K.; Naser, A. M.; Stewart, C. P.; Jannat, K.; Rahman, M. J.; Leontsini, E.; Winch, P. J.; Luby, S. P. Achieving optimal technology and behavioral uptake of single and combined interventions of water, sanitation hygiene and nutrition, in an efficacy trial (WASH Benefits) in rural Bangladesh. Trials 2018, 19 (1). DOI: 10.1186/s13063-018-2710-8

(21) Rahman, M.; Ashraf, S.; Unicomb, L.; Mainuddin, A. K. M.; Parvez, S. M.; Begum, F.; Das, K. K.; Naser, A. M.; Hussain, F.; Clasen, T.; Luby, S. P.; Leontsini, E.; Winch, P. J. WASH Benefits Bangladesh Trial: System for Monitoring Coverage and Quality in an Efficacy Trial. Trials 2018, 19 (1), 360.

(22) Unicomb, L.; Begum, F.; Leontsini, E.; Rahman, M.; Ashraf, S.; Naser, A. M.; Nizame, F. A.; Jannat, K.; Hussain, F.; Parvez, S. M.; Arman, S.; Mobashara, M.; Luby, S. P.; Winch, P. J. WASH Benefits Bangladesh Trial: Management Structure for Achieving High Coverage in an Efficacy Trial. Trials 2018, 19 (1), 359.

(23) Ercumen, A.; Pickering, A.; Kwong, L.; Mertens, A.; Arnold, B.; Benjamin-Chung, J.; Hubbard, A.; Alam, M.; Sen, D.; Islam, S.; Rahman, Z.; Kullman, C.; Chase, C.; Ahmed, R.; Parvez, S. M.; Unicomb, L.; Rahman, M.; Ram, P. K.; Clasen, T.; Luby, S. P.; Colford, J. M. Do Sanitation Improvements Reduce Fecal Contamination of Water, Hands, Food, Soil and Flies? Evidence from a Cluster-Randomized Controlled Trial in Rural Bangladesh. Environ. Sci. Technol. 2018. DOI: 10.1021/acs.est.8b02988

(24) Torondel, B.; Gyekye-Aboagye, Y.; Routray, P.; Boisson, S.; Schimdt, W.; Clasen, T. Laboratory Development and Field Testing of Sentinel Toys to Assess Environmental Faecal Exposure of Young Children in Rural India. Trans. R. Soc. Trop. Med. Hyg. 2015, 109 (6), 386-392.

(25) DiVita, M. A.; Halder, A. K.; Jahid, I. K.; Islam, M.; Sobsey, M. D.; Luby, S. P.; Ram, P. K. The utility of common household objects as markers of home hygiene in the context of access to improved sanitation. Epidemiology 2008, 19 (6), S323.

(26) Vujcic, J.; Ram, P. K.; Hussain, F.; Unicomb, L.; Gope, P. S.; Abedin, J.; Mahmud, Z. H.; Sirajul Islam, M.; Luby, S. P. Toys and Toilets: Cross-Sectional Study Using Children's Toys to Evaluate Environmental Faecal Contamination in Rural Bangladeshi Households with Different Sanitation Facilities and Practices. Trop. Med. Int. Health 2014, 19 (5), 528-536.

(27) Huda, T. M. N.; Schmidt, W.-P.; Pickering, A. J.; Mahmud, Z. H.; Islam, M. S.; Rahman, M. S.; Luby, S. P.; Biran, A. A Cross Sectional Study of the Association between Sanitation Type and Fecal 
Contamination of the Household Environment in Rural Bangladesh. Am. J. Trop. Med. Hyg. 2018, 98 (4), 967-976.

(28) Halder, A. K.; Tronchet, C.; Akhter, S.; Bhuiya, A.; Johnston, R.; Luby, S. P. Observed Hand Cleanliness and Other Measures of Handwashing Behavior in Rural Bangladesh. BMC Public Health 2010, 10 (1), 545.

(29) Pickering, A. J.; Davis, J.; Walters, S. P.; Horak, H. M.; Keymer, D. P.; Mushi, D.; Strickfaden, R.; Chynoweth, J. S.; Liu, J.; Blum, A.; Chynoweth, J. S.; Liu, J.; Blum, A.; Rogers, K.; Boehm, A. B. Hands, Water, and Health: Fecal Contamination in Tanzanian Communities with Improved, Non-Networked Water Supplies. Environ. Sci. Technol. 2010, 44 (9), 3267-3272.

(30) USEPA. Method 1604: Total Coliforms and Escherichia Coli in Water by Membrane Filtration Using a Simultaneous Detection Technique (MI Medium); US Environmental Protection Agency: Washington DC, 2002.

(31) Dufour, A. P. Escherichia Coli: The Fecal Coliform. In Bacterial indicators/health hazards associated with water; ASTM International, 1977.

(32) ISO 16649-1:2001 - Microbiology of food and animal feeding stuffs -- Horizontal method for the enumeration of betaglucuronidase-positive Escherichia coli-Part 1: Colony-count technique at 44 degrees $\mathrm{C}$ using membranes and 5-bromo-4-chloro3-indolyl beta-D-glucuronide. https://www.iso.org/standard/29823. html (accessed February 27, 2018).

(33) Nandi, B. C. The Fauna of India and the Adjacent Countrie. Diptera:Volume X, Sarcophagidae; Zoological Survey of India., 2002.

(34) Helsel, D. R. Less than Obvious - Statistical Treatment of Data below the Detection Limit. Environ. Sci. Technol. 1990, 24 (12), $1766-1774$.

(35) Gruber, S.; van der Laan, M. J. Tmle: An R Package for Targeted Maximum Likelihood Estimation, 2011.

(36) Laan, V. D.; J, M.; Polley, E. C.; Hubbard, A. E. Super Learner. Stat. Appl. Genet. Mol. Biol. 2007, 6 (1). DOI: 10.2202/15446115.1309

(37) Ahmed, K. M.; Bhattacharya, P.; Hasan, M. A.; Akhter, S. H.; Alam, S. M. M.; Bhuyian, M. A. H.; Imam, M. B.; Khan, A. A.; Sracek, O. Arsenic Enrichment in Groundwater of the Alluvial Aquifers in Bangladesh: An Overview. Appl. Geochem. 2004, 19 (2), 181-200.

(38) Ercumen, A.; Pickering, A. J.; Kwong, L. H.; Arnold, B. F.; Parvez, S. M.; Alam, M.; Sen, D.; Islam, S.; Kullmann, C.; Chase, C.; Ahmed, R.; Unicomb, L.; Luby, S. P.; Colford, J. M. Animal Feces Contribute to Domestic Fecal Contamination: Evidence from E. Coli Measured in Water, Hands, Food, Flies, and Soil in Bangladesh. Environ. Sci. Technol. 2017, 51 (15), 8725-8734.

(39) WHO Multicentre Growth Reference Study Group de Onis, M. WHO Motor Development Study: Windows of Achievement for Six Gross Motor Development Milestones. Acta Paediatr. 2006, 95, 8695.

(40) Fuller, C.; Besser, S.; Cookson, B. D.; Fragaszy, E.; Gardiner, J.; McAteer, J.; Michie, S.; Savage, J.; Stone, S. P. Technical Note: Assessment of Blinding of Hand Hygiene Observers in Randomized Controlled Trials of Hand Hygiene Interventions. Am. J. Infect. Control 2010, 38 (4), 332-334.

(41) Kwong, L. H.; Ercumen, A.; Pickering, A. J.; Unicomb, L.; Davis, J.; Luby, S. P. Hand- and Object-Mouthing of Rural Bangladeshi Children 3-18 Months Old. Int. J. Environ. Res. Public Health 2016, 13 (6), 563.

(42) Khanum, H.; Islam, M. R.; Parvin, S. Occurrences of Eggs and Larvae of Gastrointestinal Nematodes in Nails of Street Inhabitants in Dhaka City. J. Life Earth Sci. 2010, 5 (0), 75-79.

(43) Loevinsohn, B. P. Health Education Interventions in Developing Countries: A Methodological Review of Published Articles. Int. J. Epidemiol. 1990, 19 (4), 788-794.

(44) Levy, K.; Hubbard, A. E.; Eisenberg, J. N. Seasonality of Rotavirus Disease in the Tropics: A Systematic Review and MetaAnalysis. Int. J. Epidemiol. 2009, 38 (6), 1487-1496.

(45) Mounts, A. W.; Ando, T.; Koopmans, M.; Bresee, J. S.; Noel, J.; Glass, R. I. Cold Weather Seasonality of Gastroenteritis Associated with Norwalk-like Viruses. J. Infect. Dis. 2000, 181 (Supplement 2), S284-S287.

(46) Hays, B. D. Potential for Parasitic Disease Transmission with Land Application of Sewage Plant Effluents and Sludges. Water Res. 1977, 11 (7), 583-595.

(47) Black, M. I.; Scarpino, P. V.; O’Donnell, C. J.; Meyer, K. B.; Jones, J. V.; Kaneshiro, E. S. Survival Rates of Parasite Eggs in Sludge during Aerobic and Anaerobic Digestion. Appl. Environ. Microbiol. 1982, 44 (5), 1138-1143.

(48) Santamaría, J.; Toranzos, G. A. Enteric Pathogens and Soil: A Short Review. Int. Microbiol. 2003, 6 (1), 5-9.

(49) Duizer, E.; Bijkerk, P.; Rockx, B.; Groot, A. de; Twisk, F.; Koopmans, M. Inactivation of Caliciviruses. Appl. Environ. Microbiol. 2004, 70 (8), 4538-4543.

(50) Korich, D. G.; Mead, J. R.; Madore, M. S.; Sinclair, N. A.; Sterling, C. R. Effects of Ozone, Chlorine Dioxide, Chlorine, and Monochloramine on Cryptosporidium Parvum Oocyst Viability. Appl. Environ. Microbiol. 1990, 56 (5), 1423-1428.

(51) Wu, J.; Long, S. C.; Das, D.; Dorner, S. M. Are Microbial Indicators and Pathogens Correlated? A Statistical Analysis of 40 Years of Research. J. Water Health 2011, 9 (2), 265-278.

(52) Ercumen, A.; Naser, A. M.; Unicomb, L.; Arnold, B. F.; Jr, J. M. C.; Luby, S. P. Effects of Source- versus Household Contamination of Tubewell Water on Child Diarrhea in Rural Bangladesh: A Randomized Controlled Trial. PLoS One 2015, 10 (3), e0121907.

(53) Kotloff, K. L.; Nataro, J. P.; Blackwelder, W. C.; Nasrin, D.; Farag, T. H.; Panchalingam, S.; Wu, Y.; Sow, S. O.; Sur, D.; Breiman, R. F.; Faruque, A. S. G.; Saha, D.; Alonso, P. L.; Tamboura, B.; Sanogo, D.; Onwuchekwa, U.; Manna, B.; Ramamurthy, T.; Kanungo, S.; Ochieng, J. B.; Omore, R.; Oundo, J. O.; Hossain, A.; Das, S. K.; Ahmed, S.; Qureshi, S.; Quadri, F.; Adegbola, R. A.; Martin, A.; Hossain, M. J.; Akinsola, A.; Mandomando, I.; Nhampossa, T.; Acacio, S.; Biswas, S.; O’Reilly, C. E.; Mintz, E. D.; Berkeley, L. Y.; Muhsen, K.; Sommerfelt, H.; Robins-Browne, R. M.; Levine, M. M. Burden and Aetiology of Diarrhoeal Disease in Infants and Young Children in Developing Countries (the Global Enteric Multicenter Study, GEMS): A Prospective, Case-Control Study. Lancet 2013, 382 (9888), 209-222.

(54) Platts-Mills, J. A.; Babji, S.; Bodhidatta, L.; Gratz, J.; Haque, R.; Havt, A.; McCormick, B. J.; McGrath, M.; Olortegui, M. P.; Samie, A.; Shakoor, S.; Mondal, D.; Lima, I. F. N.; Hariraju, D.; Rayamajhi, B. B.; Qureshi, S.; Kabir, F.; Yori, P. P.; Mufamadi, B.; Amour, C.; Carreon, J. D.; Richard, S. A.; Lang, D.; Bessong, P.; Mduma, E.; Ahmed, T.; Lima, A. A. A. M.; Mason, C. J.; Zaidi, A. K. M.; Bhutta, Z. A.; Kosek, M.; Guerrant, R. L.; Gottlieb, M.; Miller, M.; Kang, G.; Houpt, E. R. Pathogen-Specific Burdens of Community Diarrhoea in Developing Countries: A Multisite Birth Cohort Study (MAL-ED). Lancet Glob. Health 2015, 3 (9), e564-e575.

(55) Mahmud, M. A.; Spigt, M.; Bezabih, A. M.; Pavon, I. L.; Dinant, G.-J.; Velasco, R. B. Efficacy of Handwashing with Soap and Nail Clipping on Intestinal Parasitic Infections in School-Aged Children: A Factorial Cluster Randomized Controlled Trial. PLOS Med. 2015, 12 (6), e1001837. 